\title{
Abnormal Driving Behavior Recognition Method Based on Smart Phone Sensor and CNN-LSTM
}

\author{
Hao Li \\ College of Electromechanical \\ Engineering \\ Qingdao University of Science \\ and Technology \\ Qingdao, China \\ Hanqing Wang \\ College of Electromechanical \\ Engineering \\ Qingdao University of Science \\ and Technology \\ Qingdao, China
}

\author{
Junyan Han \\ College of Electromechanical \\ Engineering \\ Qingdao University of Science \\ and Technology \\ Qingdao, China \\ Hui Xiang \\ College of Electromechanical \\ Engineering \\ Qingdao University of Science \\ and Technology \\ Qingdao, China
}

\author{
Shangqing Li \\ College of Electromechanical \\ Engineering \\ Qingdao University of Science \\ and Technology \\ Qingdao, China \\ Xiaoyuan Wang \\ College of Electromechanical \\ Engineering \\ Qingdao University of Science \\ and Technology \\ Qingdao, China
}

\begin{abstract}
Accurate identification of abnormal driving behavior is very important to improve driver safety. Aiming at the problem that threshold or traditional machine learning methods are mostly used in existing studies, it is difficult to accurately identify abnormal driving behavior of vehicles, a method of abnormal driving behavior recognition based on smartphone sensor data and convolutional neural network (CNN) combined with long and short-term memory (LSTM) was proposed. Smartphone sensors are used to collect vehicle driving data, and data sets of various driving behaviors are constructed by preprocessing the data. A recognition model based on a convolutional neural network combined with a long short-term memory network was constructed to extract depth features from data sets and recognize abnormal driving behaviors. The test results show that the accuracy of the model based on CNN-LSTM can reach $95.22 \%$, and the performance indexes can reach more than $94 \%$. Compared with the recognition model constructed only by CNN or LSTM, this model has higher recognition accuracy.
\end{abstract}

Keywords: Identification of abnormal driving behavior; Smartphone sensor; Convolutional Neural Network; Long Short-Term Memory

\section{INTRODUCTION}

With the rapid development of the automobile manufacturing industry and the improvement of people's living standards, the number of cars has continued to grow. The popularization of private cars has brought great convenience to people's daily travel and also caused a large number of traffic accidents. Expert analysis results show that driver's factors are the main factors that cause traffic accidents, and controlling driver's behaviors is an effective means to improve vehicle safety $[1,2]$. Therefore, accurate identification of abnormal driving behaviors can effectively improve driver safety and reduce the incidence of traffic accidents, which is of great significance to improving road traffic safety.

At present, there are three main methods of driving behavior recognition research by scholars at home and abroad: one is the method of driving behavior recognition based on visual images. Jain et al. used cameras to record drivers' facial features and corresponding road condition information during steering or lane change, and used a hidden Markov model to predict vehicle driving behavior [3]. Zhang et al. used vehiclemounted cameras to collect video images and proposed an interwoven deep convolutional neural network architecture for real-time and accurate recognition of driver behavior [4].
Although the method of driving behavior recognition based on visual images may produce good results in some scenes. But in practical application, this method is easily affected by light and weather and has high environmental dependence. The second is the method of driving behavior recognition based on the vehicle-mounted or external equipment. Ding et al. proposed a driver's distracted driving behavior detection method based on a frequency modulated continuous wave radar system [5]. Yao et al. collected behavioral data of taxi drivers based on onboard diagnosis system and onboard positioning system, realized initial clustering through dynamic time warping algorithm and hierarchical clustering, and put the clustering results into hidden Markov model to identify driving behaviors [6]. Wang et al. proposed a driver fatigue behavior evaluation system based on EEG signals and integrated learning methods [7]. Recognition methods based on vehicles-mounted or external devices can achieve better recognition accuracy, but the installation and arrangement of these devices are cumbersome and require a certain cost, and some devices are intrusive to the driver. The third is the method of driving behavior recognition based on smartphones sensors. Johnson et al. integrated the sensor data of the accelerometer, gyroscope, and magnetometer in the smartphone based on the dynamic time warping algorithm for 
the detection, recognition and recording of dangerous driving behaviors [8]. Xu et al. used the built-in audio sensor device of the mobile phone to realize the early recognition of the driver's distracted driving behavior [9]. Yu et al. used smartphone sensors to monitor abnormal driving behavior and adopted a support vector machine and neural network to realize fine-grained abnormal driving behavior monitoring and recognition [10]. Wu et al. collect bus acceleration, direction change and driving time data through smartphone acceleration and direction sensors, and detect and recognize abnormal bus driving behaviors based on the Naive Bayes algorithm [11]. Compared with the above two methods, the method of driving behavior recognition based on the smartphones sensor is more convenient and economical. Meanwhile, the network transmission technology and computing function of smartphones provide the possibility for further processing and in-depth analysis of the data collected in real-time. However, most of the existing research on this type of method is based on thresholds or traditional machine learning algorithms for driving behavior recognition, the recognition accuracy is not high and the generalization ability of the model is poor.

Given this, a recognition method of abnormal driving behavior based on smartphone sensors and convolutional neural network (CNN) combined with long short-term memory (LSTM) is proposed. The mobile phone sensor collects real-time vehicle driving data, the sliding window method is used to process the preprocessed time series data to generate "sample pictures". By constructing a convolutional neural network combined with a long short-term memory network model, deep feature extraction of driving behavior data and accurate recognition of abnormal driving behaviors are realized.

\section{OVERALL IDENTIFICATION METHOD FRAMEWORK}

The recognition method of vehicle abnormal driving behavior proposed in this paper mainly includes three parts: data collection, data processing, and abnormal driving behavior recognition. Firstly, the vehicle-mounted mobile phone sensor is used to collect the data of abnormal driving behavior. Then, the coordinate system of the mobile phone is adjusted and aligned with the coordinate system of the car body, and the obtained sensor parameters are corrected to the coordinate system parallel to the car body, and data filtering and normalization processing are carried out successively. Finally, the driving behavior data is divided into $\mathrm{N}$ "sample pictures" by the sliding window method, input into the convolutional neural network for deep feature extraction, and the extracted deep features are sent to the long short-term memory network for abnormal driving behavior recognition. The framework of the overall recognition method is shown in Figure 1.

\section{EXPERIMENTAL DATA COLLECTION AND PROCESSING}

\subsection{Smart Phone Sensor Data Collection}

To analyze the movement characteristics of the vehicle in different driving conditions, smartphone sensors are used to collect vehicle dynamic data, mainly including acceleration sensor data $A_{x}, A_{y}, A_{z}$, which represent the acceleration of the phone's X-axis, Y-axis, and Z-axis; gyroscope sensor data $G_{x}, G_{y}, G_{z}$, which represent the angular velocity around the $\mathrm{X}$-axis, $\mathrm{Y}$-axis, and $\mathrm{Z}$-axis of the mobile phone; the mobile phone attitude angle obtained by fusing the magnetic field sensor data and the acceleration sensor data: pitch, roll, yaw, Where pitch is the angle between the positive direction of the $\mathrm{Y}$-axis and the horizontal direction when the phone rotates around the $\mathrm{X}$-axis, the roll is the angle between the positive direction of the $\mathrm{X}$-axis and the horizontal direction when the phone rotates around the Y-axis, and yaw is the angle when the phone rotates around the $\mathrm{Z}$-axis, The angle between the positive direction of the $\mathrm{Y}$-axis and the north direction of the magnetic field.

Before data collection, the mobile phone is first placed in the car with a bracket to make the mobile phone face up and the $\mathrm{Y}$-axis is parallel to the front of the car. The coordinate system of the mobile phone is aligned with the coordinate system of the car body, which is called "standard posture" [12], as shown in Figure 2. Since the vehicle will produce different motion states during the driving process, it cannot be guaranteed that the vehicle-mounted mobile phone will be placed in strict accordance with the standard posture. Therefore, it is necessary to perform real-time rotation correction on the vehicle-mounted mobile phone sensor data and rotate it to the data in the "standard attitude" shown in Figure 2.

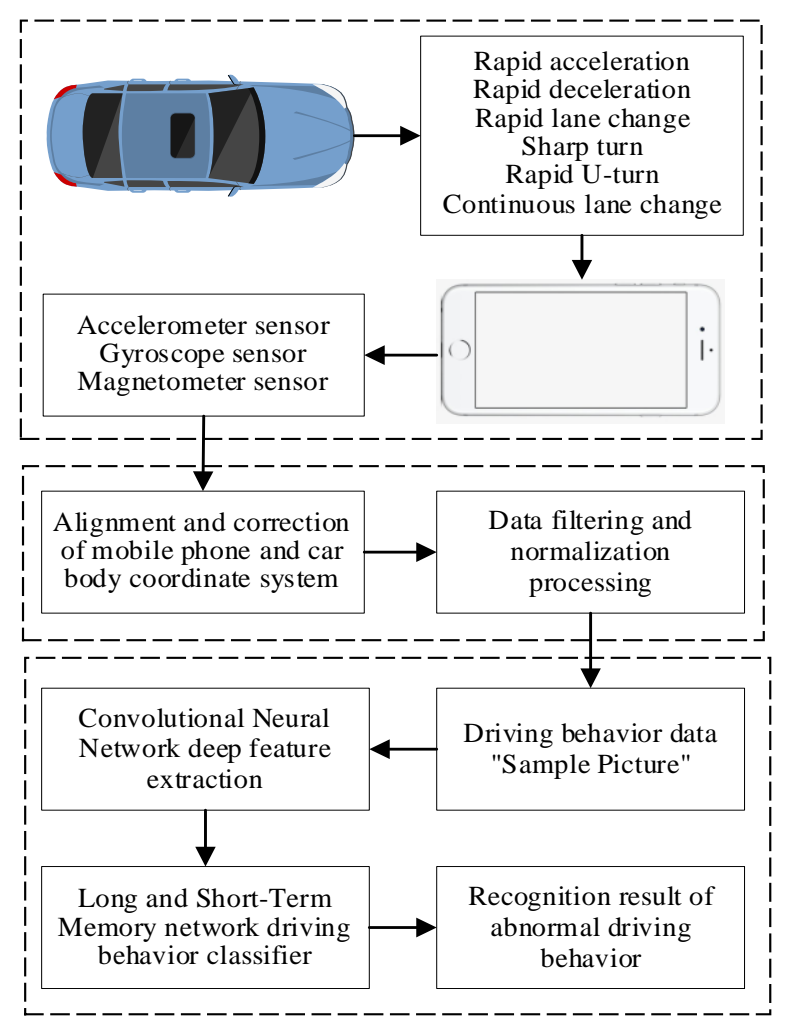

Figure 1 The framework of the overall recognition method 


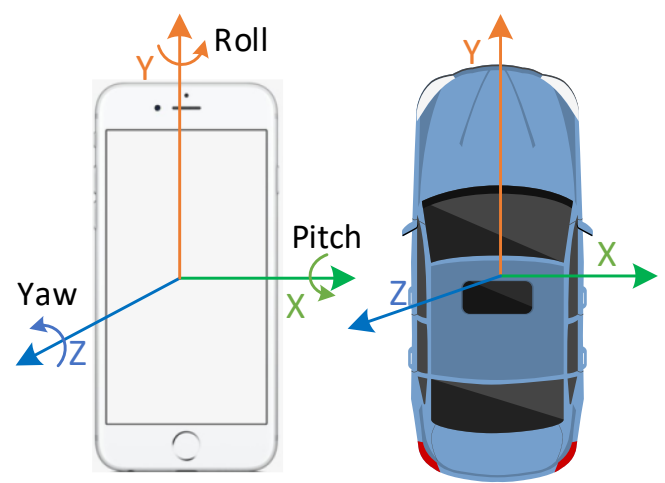

Figure 2 Coordinate alignment

The conversion of the three-axis acceleration and angular velocity in the non-standard posture into the corresponding data under the standard posture is regarded as a threedimensional coordinate rotation problem, which is decomposed into the superposition of the three coordinate axis rotations, and then converted into a matrix transformation problem [12]. In this paper, the most commonly used method of coordinate axis rotation: "yaw-pitch-roll" method, the rotation formula to convert the non-standard posture coordinate system to the standard posture coordinate system can be obtained by formula (1). The sensor data collected under a non-standard attitude can be converted to the corresponding sensor data under standard attitude by using Formula (2).

$$
R=R_{Z}(\varphi) R_{x}(\alpha) R_{y}(\beta)=
$$

$$
\begin{gathered}
{\left[\begin{array}{ccc}
\cos \varphi & \sin \varphi & 0 \\
-\sin \varphi & \cos \varphi & 0 \\
0 & 0 & 1
\end{array}\right]\left[\begin{array}{ccc}
1 & 0 & 0 \\
0 & \cos \alpha & \sin \alpha \\
0 & -\sin \alpha & \cos \alpha
\end{array}\right]\left[\begin{array}{ccc}
\cos \beta & 0 & -\sin \beta \\
0 & 1 & 0 \\
\sin \beta & 0 & \cos \beta
\end{array}\right]=} \\
{\left[\begin{array}{ccc}
R_{11} & \sin \varphi \cos \alpha & R_{13} \\
R_{21} & \cos \varphi \cos \alpha & R_{23} \\
\cos \alpha \sin \beta & -\sin \alpha & \cos \alpha \cos \beta
\end{array}\right]} \\
X^{\prime}=R X
\end{gathered}
$$

Where $R_{x}(\alpha)$ is the $\mathrm{X}$-axis rotation matrix, $R_{y}(\beta)$ is the $\mathrm{Y}$-axis rotation matrix, and $R_{Z}(\varphi)$ is the Z-axis rotation matrix,

$R_{11}=\cos \varphi \cos \beta+\sin \varphi \sin \alpha \sin \beta$,

$R_{21}=-\sin \varphi \cos \beta+\cos \varphi \sin \alpha \sin \beta$,

$R_{13}=-\cos \varphi \sin \beta+\sin \varphi \sin \alpha \cos \beta$,

$R_{23}=\sin \varphi \sin \beta+\cos \varphi \cos \alpha \cos \beta$.

When the mobile phone coordinate system is aligned with the vehicle coordinate system, and data rotation correction is performed, the movement state of the vehicle body can be characterized by the data of the built-in sensor of the smartphone. To analyze the dynamic parameter characteristics of the vehicle-mounted mobile phone under different abnormal driving behaviors of the vehicle, the sensors data of the mobile phone under the seven motion states of the vehicle's rapid acceleration, rapid deceleration, rapid lane change, sharp turn, rapid U-turn, continuous lane change and normal driving are collected. The experimental data contains 8 characteristic variables, including three-axis acceleration data $A_{x}, A_{y}, A_{z}$ and three-axis combined acceleration $A_{s}$; three-axis angular velocity data $G_{x}, G_{y}, G_{z}$, and three-axis combined angular velocity $G_{s}$. The calculation formulas of three-axis combined acceleration $A_{s}$ and three-axis combined angular velocity $G_{s}$ are as follows.

$$
\begin{aligned}
A_{s} & =\sqrt{A_{x}^{2}+A_{y}^{2}+A_{z}^{2}} \\
G_{s} & =\sqrt{G_{x}^{2}+G_{y}^{2}+G_{z}^{2}}
\end{aligned}
$$

During the experimental data collection process, the time when the specific abnormal driving behavior occurred and the corresponding tags were artificially recorded. Normal driving, rapid acceleration, rapid deceleration, rapid lane change, sharp turn, rapid U-turn, and continuous lane change are represented by 0-6 labels respectively.

\subsection{Data Processing}

In the process of experimental data collection, due to the complex road conditions, the vehicle's own vibration and sensor accuracy, a certain amount of high-frequency noise will appear in the collected raw sensor data. Therefore, before analyzing the variation characteristics of various abnormal driving behaviors in acceleration and direction angle, the collected original data should be filtered to eliminate highfrequency noise. In this paper, a sliding mean filter algorithm is used to smooth and filter the sensor data, and the mean filter expression is shown in the formula:

$$
\bar{X}_{n}=\frac{1}{M} \sum_{i=0}^{M-1} X_{n-i}
$$

Where $M$ is the sliding filter window size, and $X_{n-1}$ is the $n-i$ th original data. The filter window $M$ will have a greater impact on the filtering effect. According to the original data collection frequency $(50 \mathrm{~Hz})$, we choose $M=10$ to smooth the original data.

Through the analysis of the filtered sensor data, it is found that the dimension of each sensor feature data is different, which easily affects the target result. Therefore, the original sensor feature data is normalized to map the result value of the feature data to $[0,1]$. The function is shown in Equation 6.

$$
a^{\prime}=\frac{a-\min a}{\max a-\min a}
$$

Where $a$ is the original data, and $a^{\prime}$ represents the normalized value of the input sample $a$.

\section{MODEL}

\subsection{Convolutional Neural Network (CNN)}

$\mathrm{CNN}$ is one of the most important networks in the field of deep learning. $\mathrm{CNN}$ is a feedforward neural network that can extract features from data with a convolutional structure [13]. Its basic structure consists of an input layer, a convolutional layer, a pooling layer, a fully connected layer, and an output layer [14]. The convolutional layer performs deep feature 
extraction on the original data through local connections, meanwhile reducing the original data dimension. The pooling layer is also called the down-sampling layer, which can effectively reduce the complexity of the network, reduce the number of training parameters, and play the role of secondary feature extraction and dimensionality reduction. The fully connected layer can integrate the local information with classification in the convolutional layer or the pooling layer. The basic network structure of CNN is shown in Figure 3 .

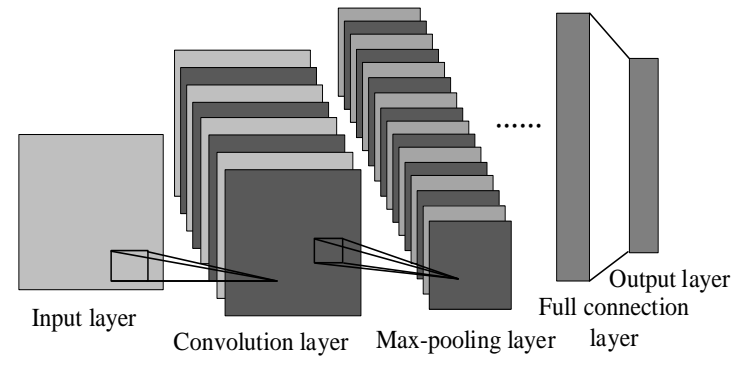

Figure 3 Basic network structure of the convolutional neural network

\subsection{Long Short-Term Memory (LSTM)}

In order to solve the long-term dependency problem of Recurrent Neural Network (RNN) [15], Hochreiter et al. proposed an improved RNN in 1997 [16], that is, long shortterm memory (LSTM), which uses a unique gate structure to effectively solve the problem of gradient explosion and gradient disappearance in RNN. LSTM has shown excellent performance in the recognition and prediction of time series data. Therefore, in this study, LSTM was selected to analyze the driving behavior characteristic data with time series.

Each layer of the LSTM network is composed of cyclically linked unit blocks, which mainly include three gate structures: forget gates, input gates, and output gates [17]. The network unit block structure of this study is shown in Figure 4. The forgetting gate determines how much information about the memory cell state $C_{t-1}$ at the previous moment is retained; the input gate updates the characteristic information according to $h_{t-1}$ and $X_{t}$, and combines it with the memory cell state $C_{t-1}$ at the previous moment. It is the new memory cell state $C_{t}$; the output gate determines how much current information can be output.

The calculation formula of the LSTM neuron is as follows:

$$
\begin{gathered}
f_{t}=\sigma\left(W_{f} \cdot\left[h_{t-1}, X_{t}\right]+b_{f}\right) \\
i_{t}=\sigma\left(W_{i} \cdot\left[h_{t-1}, X_{t}\right]+b_{i}\right) \\
\hat{C}_{t}=\tanh \left(W_{c} \cdot\left[h_{t-1}, X_{t}\right]+b_{c}\right) \\
C_{t}=f_{t} * C_{t-1}+i_{t} * \hat{C}_{t} \\
o_{t}=\sigma\left(W_{o} \cdot\left[h_{t-1}, X_{t}\right]+b_{o}\right) \\
h_{t}=o_{t} * \tanh \left(C_{t}\right)
\end{gathered}
$$

Where $f_{t}, i_{t}, o_{t}$ are forget gate, input gate, and output gate respectively, $X_{t}, \hat{C}_{t}, h_{t-1}, h_{t}$ are input value, candidate state value, previous hidden layer output, this hidden layer output respectively, $W_{f}, W_{i}, W_{c}, W_{o}$ are the weights of the corresponding gates respectively, $b_{f}, b_{i}, b_{c}, b_{o}$ are the biases of the corresponding gates respectively, $\sigma$ represents the sigmoid activation function, and tanh represents the nonlinear activation function.

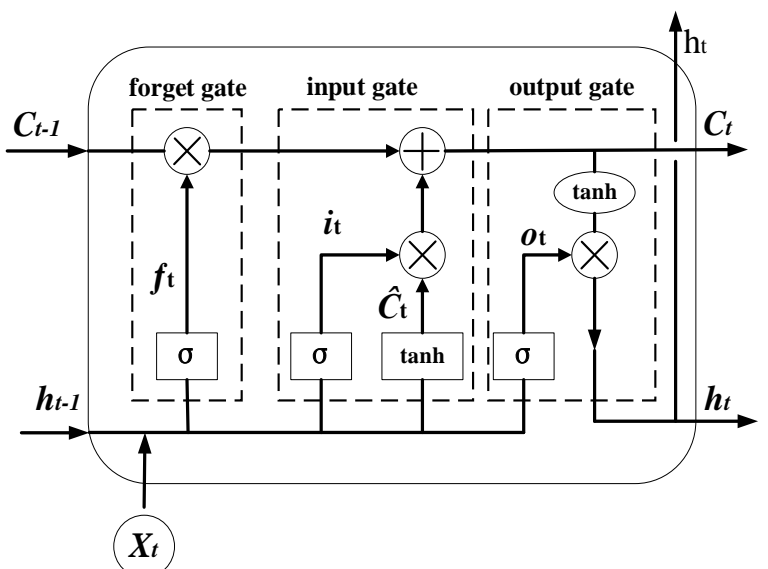

Figure 4 Unit structure of LSTM network

\subsection{Recognition Model of Vehicle Abnormal Driving Behavior Based on CNN-LSTM}

Taking into account the advantages of convolutional neural networks and long short-term memory networks in feature extraction and processing dynamic timing information, this paper constructs a recognition model of vehicle abnormal driving behavior based on CNN and LSTM. The basic framework is shown in Figure 5. First, use the sliding window method to divide the smartphone sensor data into time series, construct a two-dimensional driving behavior feature data "sample picture" and transmit it to the input layer of the convolutional neural network; through the convolutional layer, the deeper features in the original data are processed. Extract and use the pooling layer to perform feature dimensionality reduction, reduce the complexity of the network, and finally obtain the optimal driving behavior feature parameters. Then, LSTM was used to construct the model and analyze the depth characteristic parameters extracted by CNN on time series. Finally, Softmax classifier is used to classify and identify driving behaviors, which are divided into seven categories: rapid acceleration, rapid deceleration, rapid lane change, sharp turn, rapid U-turn, continuous lane change and normal driving. 


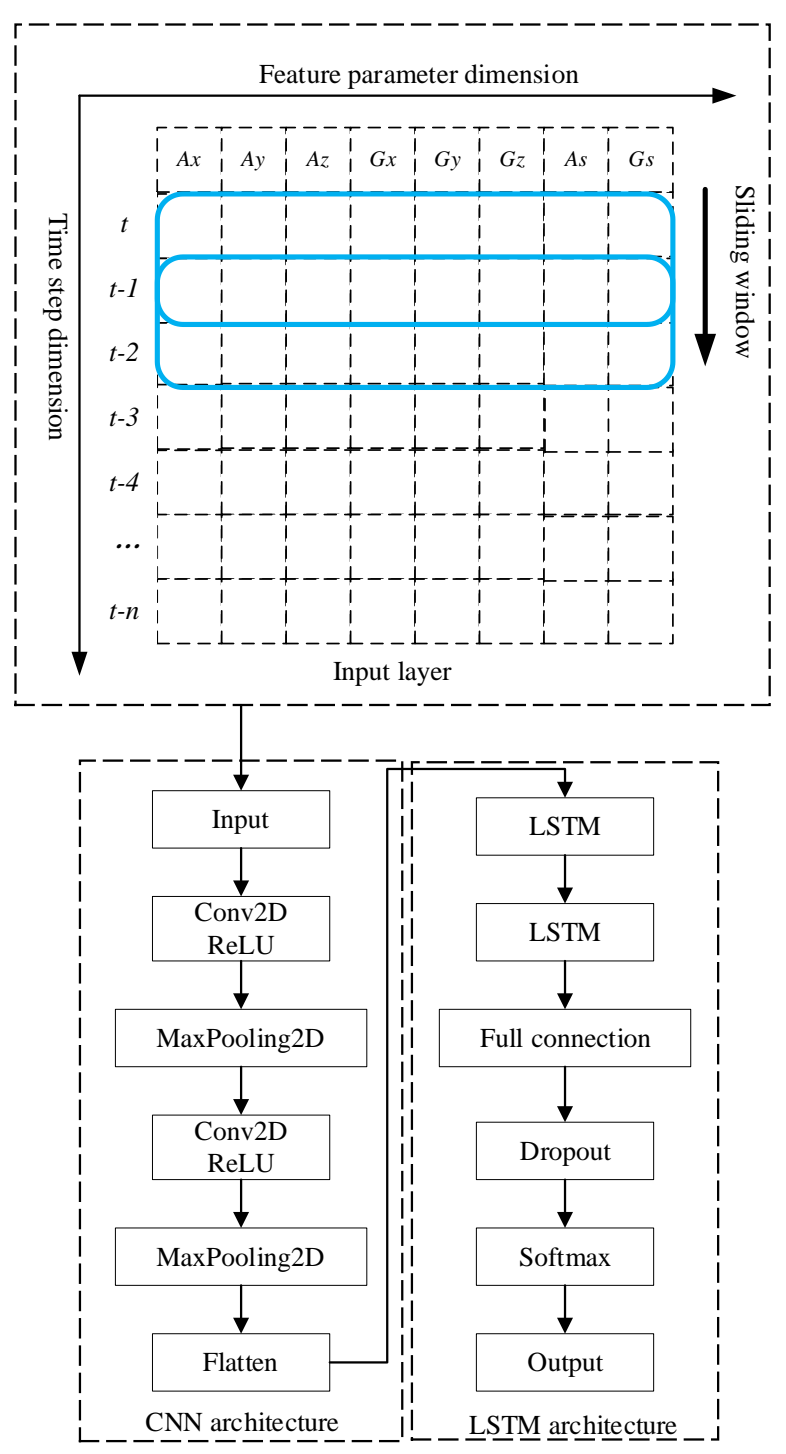

Figure 5 Basic framework of abnormal driving behavior recognition model based on CNN-LSTM

(1) Input layer. The pre-processed driving behavior data: $X$ axis acceleration $A_{x}, \mathrm{Y}$-axis acceleration $A_{y}, \mathrm{Z}$-axis acceleration $A_{z}, \mathrm{X}$-axis angular velocity $G_{x}, \mathrm{Y}$-axis angular velocity $G_{y}$, Z-axis angular velocity $G_{z}$, triaxial acceleration $A_{s}$ and triaxial angular velocity $G_{s}$ were connected in series into the time series feature vectors of 8 dimensions. According to the time series, the data per second is expressed as a two-dimensional matrix of "time step $\times$ feature vector", and the "sample picture" of driving behavior data is generated on the data set by sliding window in accordance with the data division method of input layer in the above figure. The sensor data (acquisition frequency is $50 \mathrm{~Hz}$ ) is traversed with a 2second sliding window and a 1 -second sliding step, and $\mathrm{N}$ sample pictures of " $100 \times 8$ " pixels are generated. Where 100 is 100 sensor data collected within 2 seconds, 8 is the dimension of the input feature vector, and the number of sample pieces is N.
(2) CNN architecture. The processed "sample pictures" of the input layer are sent to the convolutional neural network, and $\mathrm{CNN}$ is used for feature extraction. The CNN architecture consists of an input layer, two convolutional layers, two pooling layers and a flatten layer. In the two convolutional layers, the number of convolution kernels is 16,32 , and the size is $3 \times 3$. The step size is 1 in both the time step dimension and the feature parameter dimension. A filling is used, and the ReLU activation function is selected for activation. In the two pooling layers, the pooling core size is $2 \times 2$, the step size is 2 in both the time step dimension and the feature parameter dimension, and the maximum pooling principle is selected. In order to alleviate the over-fitting phenomenon and speed up the training speed of the neural network, a Batch Normalization layer is added after each layer of the convolutional layer. Finally, through the Flatten operation, the deep abstract features extracted are converted into global feature vectors as the input of the LSTM layer.

(3) LSTM architecture. LSTM is used to train the global feature variables extracted by CNN. After adjustment and testing, it can be known that the 2-layer LSTM structure achieves the best prediction effect. The number of LSTM neurons is 64 and 128 respectively. The number of neurons in the fully connected layer is also set to 128 . The dropout layer has a loss rate of 0.5 . The role of this layer is to alleviate the over-fitting phenomenon of the model. Finally, the probability of each driving behavior category is output through the Softmax activation function of the output layer.

\section{EXPERIMENT AND ANALYSIS}

\subsection{Model Training}

In this study, a neural network model was built based on Keras deep learning framework and Python3.7 programming language. The experimental platform configuration is: Intel(R)Core(TM) i7-10870H CPU @ 2.20GHz, memory for 16G, graphics card for GTX3060 Laptop, video memory for $6 \mathrm{G}$.

The total number of original data samples selected in the experiment is 121,000 . After the combined screening, 2400 "sample pictures" are generated, which including 1200 normal driving data, rapid acceleration data, rapid deceleration data, rapid lane change data, sharp turn data, rapid U-turn data and continuous lane change data are 200 sheets each. The data set is divided into training set and test set according to the ratio of $7: 3$. The identification of abnormal driving behavior in this study is a multi-classification problem, so the label needs to be One_hot encoded first.

After the initial network model is constructed, the network parameters need to be set and adjusted before the model is trained. In this paper, the Adam optimization algorithm is selected to adjust and optimize the model parameters. At the same time, in order to prevent the model from falling into a local minimum, the learning rate is set to 0.001 , and the number of iterations of the model epochs is set to 1000 . The model's accuracy rate change and loss function value convergence during the training process are shown in Figures 6 and 7. It can be seen from Figure 6 that the recognition accuracy of the CNN-LSTM model can reach more than $95 \%$, achieving high recognition accuracy. The change of the loss function can also reflect the training effect of the algorithm model. It can be seen from Figure 7 that as the number of training increases, the loss function of the CNN-LSTM model 
converges faster. After 300 epoch, the convergence tends to be flat, and the loss value is close to 0.04 , with better identification performance.

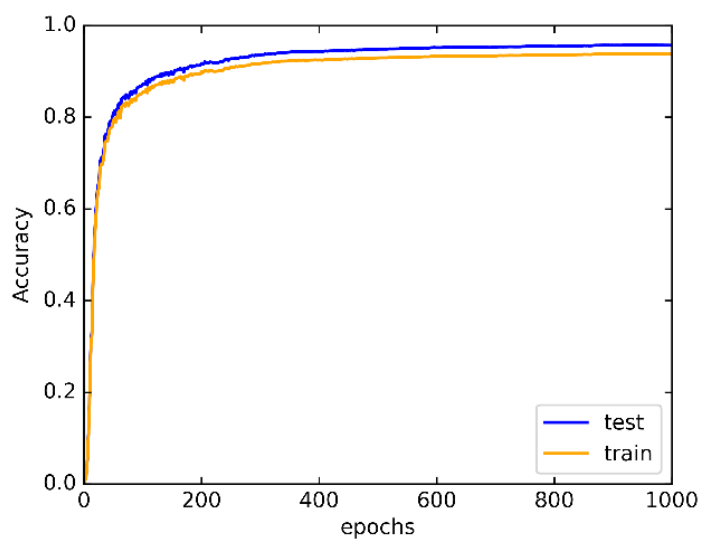

Figure 6 The recognition accuracy of the CNN-LSTM model

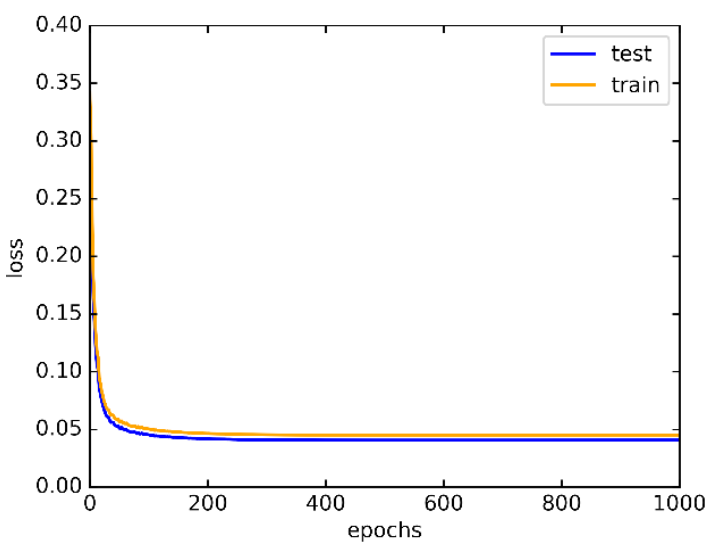

Figures 7 Loss change curve of CNN-LSTM model

\subsection{Model Testing and Comparison}

In order to evaluate the overall performance of the $\mathrm{CNN}$ LSTM model for the recognition of abnormal driving behaviors, all types of abnormal driving behaviors are regarded as one type, and only one type of driving behavior is identified whether it is abnormal or normal. We define the evaluation indicators of the following models according to True Positive (TP), True Negative (TN), False Positive (FP), and False Negative (FN) [18]:

Accuracy: reflects the accuracy of the model's recognition of various driving behaviors, that is, the ratio of the number of samples correctly classified by the classifier to the total number of samples.

$$
\text { Accuracy }=\frac{T P+T N}{T P+T N+F P+F N}
$$

Precision: reflects the proportion of real A-type driving behavior in the sample predicted by the model to be A-type driving behavior.

$$
\text { Precision }=\frac{T P}{T P+F P}
$$

Recall: reflects the model's proportion of all Class A driving behavior samples recognized as Class $\mathrm{A}$.

$$
\operatorname{Re} \text { call }=\frac{T P}{T P+F N}
$$

F1-Score: A comprehensive indicator of the balance between accuracy and recall, which is a weighted average of model accuracy and recall.

$$
\mathrm{F}-1=\frac{2 \cdot \text { precision } \cdot \text { recall }}{\text { precision }+ \text { recall }}
$$

The identification results of the model were calculated and analyzed according to the above formula. The overall identification accuracy of the model was $95.22 \%$, and the details of other evaluation indicators of the model were shown in Table 1. The model has a better recognition effect on all abnormal driving behaviors, and the Recall can reach $95.83 \%$. All the performance indexes of normal driving behaviors and abnormal driving behaviors identified by the model are more than $94 \%$, and the F1-Score performance indicators exceeded $95 \%$. The model achieved good recognition results.

Table 1 Various evaluation indicators of CNN-LSTM model

\begin{tabular}{|c|c|c|c|}
\hline $\begin{array}{c}\text { Evaluation } \\
\text { index }\end{array}$ & $\begin{array}{c}\text { Precision } \\
(\boldsymbol{\%})\end{array}$ & $\begin{array}{c}\text { Recall } \\
(\boldsymbol{\%})\end{array}$ & $\begin{array}{c}\text { F1-Score } \\
(\boldsymbol{\%})\end{array}$ \\
\hline $\begin{array}{c}\text { Normal driving } \\
\text { behavior }\end{array}$ & 95.79 & 94.72 & 95.25 \\
\hline $\begin{array}{c}\text { Abnormal driving } \\
\text { behavior }\end{array}$ & 94.78 & 95.83 & 95.31 \\
\hline
\end{tabular}

In order to further evaluate the accuracy of the model's recognition of the six abnormal driving behaviors, 80 sets of data for each of rapid acceleration (RA), rapid deceleration (RD), sharp turning (ST), rapid lane change (RLC), rapid Uturn (RU), and continuous lane change (CLC) driving behavior were selected to construct a new test set and input them respectively the trained CNN-LSTM model is recognized. The recognition results are shown in Table 2.

Table 2 Recognition accuracy rate of six abnormal driving behaviors

\begin{tabular}{|c|c|c|c|}
\hline driving behavior & RA & RD & ST \\
\hline Accuracy (\%) & 96.25 & 97.5 & 93.75 \\
\hline driving behavior & RLC & RU & CLC \\
\hline Accuracy (\%) & 95 & 92.5 & 93.75 \\
\hline
\end{tabular}

It can be seen from Table 2 that the accuracy of the model for the recognition of six abnormal driving behaviors exceeds $92 \%$, and a good recognition effect is achieved. Among them, the recognition accuracy of rapid acceleration and rapid deceleration driving behavior can reach more than $96 \%$, and the recognition effect is the best. Because the data change lasts for a short time, the driving behavior can be completed within the sliding partition window time, so the recognition accuracy is high. Second, the changes time of driving behavior data of rapid U-turns, sharp turns, and continuous lane changes have a relatively long duration. The driving behavior may not be completed within the sliding partition 
window, which will reduce the accuracy of the model's recognition.

In order to highlight the effectiveness and superiority of the CNN-LSTM abnormal driving behavior recognition model proposed in this paper, CNN and LSTM are used to process the same data samples respectively, and the abnormal driving behavior recognition model is established for comparison. In order to reduce the chance of experimental results, the same data set was used to train each network model for three times respectively, and the recognition results of the model, such as overall recognition Accuracy, recognition Precision of abnormal driving behavior, Recall of abnormal driving behavior and F1-Score, a comprehensive index of abnormal driving behavior, were averaged. The evaluation indicators of each model are shown in Table 3.

Table 3 Evaluation index of each model

\begin{tabular}{|c|c|c|c|c|}
\hline $\begin{array}{c}\text { Evaluation } \\
\text { index }\end{array}$ & $\begin{array}{c}\text { Accuracy } \\
(\boldsymbol{\%})\end{array}$ & $\begin{array}{c}\text { Precision } \\
(\boldsymbol{\%})\end{array}$ & $\begin{array}{c}\text { Recall } \\
(\boldsymbol{\%})\end{array}$ & $\begin{array}{c}\text { F1-Score } \\
(\boldsymbol{\%})\end{array}$ \\
\hline CNN & 91.21 & 90.38 & 92.22 & 91.29 \\
\hline LSTM & 92.82 & 92.01 & 93.79 & 92.89 \\
\hline $\begin{array}{c}\text { CNN- } \\
\text { LSTM }\end{array}$ & 95.65 & 95.14 & 96.21 & 95.67 \\
\hline
\end{tabular}

It can be seen from Table 3 that the recognition accuracy and comprehensive performance indicators of the LSTM model can reach about 93\%. Compared with the CNN model, various performance indicators perform better. The CNNLSTM model integrates the advantages of CNN depth feature extraction and LSTM for dynamic time-series information processing. Compared with the LSTM model, the recognition accuracy is increased by about $3 \%$, and various performance indicators for abnormal driving behavior recognition are improved.

\section{CONCLUSION}

Accurate identification of abnormal driving behavior is of great significance to improve road traffic safety. Aiming at the problems of the poor accuracy of the abnormal driving behavior recognition method and the poor generalization ability of the model in the existing research. This paper proposes an abnormal driving behavior recognition method based on smartphone sensors and CNN-LSTM. The vehiclemounted smartphone sensor data is used to characterize the movement characteristics of each driving behavior of the vehicle, and the acceleration and angular velocity sensor data are collected, and the driving behavior characteristic data set is constructed through the sensor data coordinate correction, data filtering, and data normalization processing. Given the advantages of CNN and LSTM networks in feature extraction and processing of dynamic timing information, a recognition model of vehicle abnormal driving behavior based on CNN and LSTM is constructed, and the effectiveness and accuracy of the model are verified by using feature data sets. Compared with the CNN and LSTM models, the results show that the performance indicators of the model have been improved and have higher recognition accuracy.

\section{ACKNOWLEDGMENTS}

This study was funded by the Qingdao Top Talent Program of Entrepreneurship and Innovation (Grant No.19-3-2-11-zhc), the Natural Science Foundation of Shandong Province (Grant No. ZR2020MF082), the Foundation of Shandong Intelligent Green Manufacturing Technology and Equipment Collaborative Innovation Center (Grant No. IGSD-2020-012), and the National Key Research and Development Project (Grant No.2018YFB1601500).

\section{REFERENCES}

[1] Chan, T. K., Chin, C. S., Chen, H., \& Zhong, X. (2019). A comprehensive review of driver behavior analysis utilizing smartphones. IEEE Transactions on Intelligent Transportation Systems, 21(10), 4444-4475.

[2] Ellison, A. B., Greaves, S. P., \& Bliemer, M. C. (2015). Driver behaviour profiles for road safety analysis. Accident Analysis \& Prevention, 76, 118-132.

[3] Jain, A., Koppula, H. S., Raghavan, B., Soh, S., \& Saxena, A. (2015). Car that knows before you do: Anticipating maneuvers via learning temporal driving models. In Proceedings of the IEEE International Conference on Computer Vision (pp. 3182-3190).

[4] Zhang, C., Li, R., Kim, W., Yoon, D., \& Patras, P. (2020). Driver behavior recognition via interwoven deep convolutional neural nets with multi-stream inputs. IEEE Access, 8, 191138-191151.

[5] Ding, C., Chae, R., Wang, J., Zhang, L., Hong, H., Zhu, X., \& Li, C. (2019). Inattentive driving behavior detection based on portable FMCW radar. IEEE Transactions on Microwave Theory and Techniques, 67(10), 4031-4041.

[6] Yao, Y., Zhao, X., Wu, Y., Zhang, Y., \& Rong, J. (2021). Clustering driver behavior using dynamic time warping and hidden Markov model. Journal of Intelligent Transportation Systems, 25(3), 249-262.

[7] Wang, P., Min, J., \& Hu, J. (2018). Ensemble classifier for driver's fatigue detection based on a single EEG channel. IET Intelligent Transport Systems, 12(10), 1322-1328.

[8] Johnson, D. A., \& Trivedi, M. M. (2011, October). Driving style recognition using a smartphone as a sensor platform. In 2011 14th International IEEE Conference on Intelligent Transportation Systems (ITSC) (pp. 16091615). IEEE.

[9] Xu, X., Yu, J., Chen, Y., Zhu, Y., Qian, S., \& Li, M. (2017). Leveraging audio signals for early recognition of inattentive driving with smartphones. IEEE Transactions on Mobile Computing, 17(7), 1553-1567.

[10] Yu, J., Chen, Z., Zhu, Y., Chen, Y., Kong, L., \& Li, M. (2016). Fine-grained abnormal driving behaviors detection and identification with smartphones. IEEE transactions on mobile computing, 16(8), 2198-2212.

[11] Wu, X., Zhou, J., An, J., \& Yang, Y. (2018, March). Abnormal driving behavior detection for bus based on the Bayesian classifier. In 2018 Tenth International Conference on Advanced Computational Intelligence (ICACI) (pp. 266-272). IEEE. 
[12] Wang, R., Xie, F., Zhao, J., Zhang, B., Sun, R., \& Yang, J. (2020). Smartphone Sensors-Based Abnormal Driving Behaviors Detection: Serial-Feature Network. IEEE Sensors Journal.

[13] Li, Z., Liu, F., Yang, W., Peng, S., \& Zhou, J. (2021). A survey of convolutional neural networks: analysis, applications, and prospects. IEEE Transactions on Neural Networks and Learning Systems.

[14] LeCun, Y., Bottou, L., Bengio, Y., \& Haffner, P. (1998). Gradient-based learning applied to document recognition. Proceedings of the IEEE, 86(11), 22782324.
[15] Bengio, Y., Simard, P., \& Frasconi, P. (1994). Learning long-term dependencies with gradient descent is difficult. IEEE transactions on neural networks, 5(2), 157-166.

[16] Hochreiter, S., \& Schmidhuber, J. (1997). Long shortterm memory. Neural computation, 9(8), 1735-1780.

[17] Jia, S., Hui, F., Li, S., Zhao, X., \& Khattak, A. J. (2020). Long short-term memory and convolutional neural network for abnormal driving behaviour recognition. IET Intelligent Transport Systems, 14(5), 306-312.

[18] Khodairy, M. A., \& Abosamra, G. (2021). Driving Behavior Classification Based on Oversampled Signals of Smartphone Embedded Sensors Using an Optimized Stacked-LSTM Neural Networks. IEEE Access, 9, 49574972. 